\title{
Extracellular DNA (eDNA) enables early detection of the phenotypic switch of Pseudomonas sp. during biofilm development
}

\author{
Fatemeh Bajoul Kakahi', Dingrong Kang ${ }^{1}$, Daniel C. Volke ${ }^{2}$, Nicolas T. Wirth ${ }^{2}$, Pablo I. \\ Nikel², Frank Delvigne $^{1^{*}}$
}

${ }^{1}$ Terra Research and Teaching Centre, Microbial Processes and Interactions (MiPI), Gembloux Agro-Bio Tech, University of Liege, Gembloux, Belgium.

${ }^{2}$ The Novo Nordisk Foundation Center for Biosustainability, Technical University of Denmark, 2800 Kongens Lyngby, Denmark.

*Corresponding author: F.Delvigne@uliege.be

13

\section{Abstract}

Microbial populations undergo phenotypic switching as a response to environmental perturbations. For instance, some bacteria switch from a planktonic lifestyle to biofilm, resulting in altered physiological properties such as increased robustness depending on the conditions. However, the precise detection of phenotypic switching events during the bacterial life cycle is still a technical challenge. Propidium iodide (PI) is one of the most frequently used fluorescence indicators for assessing cell viability based on membrane permeability, yet PI-stained cells sometimes display a red-but-not-dead phenotype. In Escherichia coli, this phenomenon is connected to modulation of porins in the outer membrane (OM) to adapt OM permeability according to nutrient availability. In this study, we explored PI staining to assess phenotypic changes in Pseudomonas sp. during biofilm development. We show that this switch is linked to excretion of extracellular DNA (eDNA), rather than modification of OM permeability. Confocal laser scanning microscopy (CLSM) enabled direct visualization of red fluorescent clusters outside intact membranes of viable cells, suggesting that PI binds eDNA. Besides, the occurrence of PI-positive sub-populations was correlated with biofilm formation in the model bacterium Pseudomonas putida KT2440. Engineered derivatives thereof with altered biofilm-forming capabilities exposed a whole 
continuum of phenotypic states involving planktonic cells and aggregates, and were identified

2 according to the dynamic change of PI-positive cells with flow cytometry analysis. Our results

3 demonstrate that PI is a fast, convenient and versatile staining for eDNA to rapidly monitor the

4 phenotypic switching of Pseudomonas sp. during transitions in the bacterial lifestyle.

5

6 Graphical abstract

10

11

12

13

14

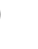

6

\section{\>>>>>> Population State}
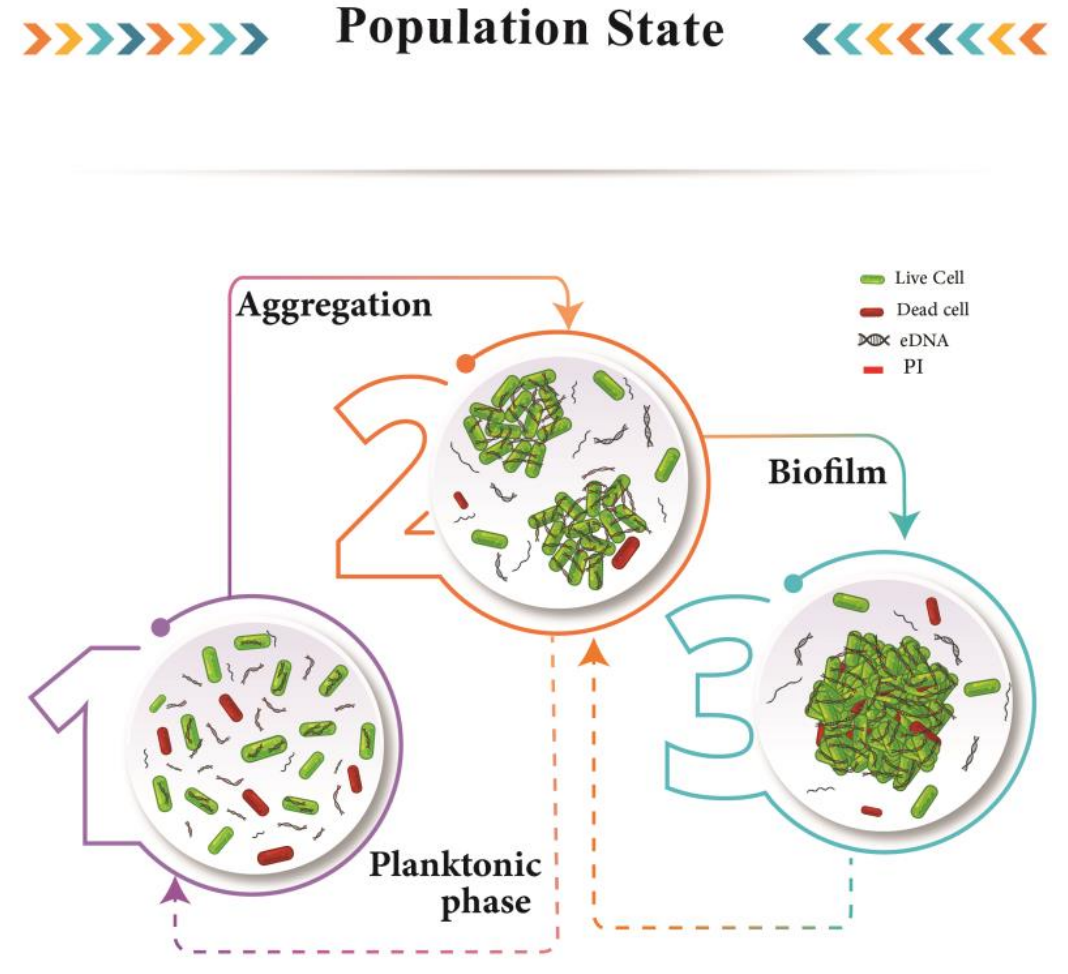

5 Running title: Tracking eDNA in Pseudomonas phenotypic switch

7 Keywords: Propidium iodide (PI), Flow cytometry, Extracellular DNA (eDNA), Biofilm, Aggregation, Sub-population, Phenotypic switching 


\section{Introduction}

3 In natural ecosystems, many bacteria are able to switch between two modes of growth: on the one hand, a unicellular lifestyle, in which the cells are swimming freely, also called planktonic, and on the other hand, a multicellular lifestyle, in which the cells are sessile and form biofilms (Berlanga and Guerrero, 2016). Phenotypic switching between these two states is an important phenomenon involved in evolutionary development of bacteria and contributing to single-cell, as well as whole population fitness (Sousa et al., 2011). While bacteria convert to a sessile state, the expression of several genes is altered causing a phenotype distinguishable from planktonic growing cells (Sousa et al., 2011). Many microbial processes are dependent on phenotypic switching (e.g., persistence, utilization of alternative carbon source, competence...), leading to the formation of a heterogeneous, diverse and dynamic population that can overcome stresses and colonize new environments (Schreiber et al., 2016)(Ackermann, 2015)(Eldar and Elowitz, 2010)(Delvigne et al., 2014). Biofilm formation is indeed an efficient microbial strategy for survival. Upon switching from planktonic to biofilm mode of growth, bacteria go through several complex physiological, metabolic, and phenotypic diversifications (Drenkard, 2003). During biofilm growth, bacteria adopt a biofilm-specific phenotype, fundamentally different from that expressed in the corresponding planktonic cells. This switch to the biofilm-specific phenotype can trigger mechanisms responsible for antimicrobial resistance, enhanced virulence, and persistence (Mah and O'Toole, 2001). Our understanding of phenotypic switching in the biofilm field is still limited. However, it is critical to understand how phenotypic switching leads to the formation of biofilms, the understanding of which has important consequences from both a fundamental (i.e., microbial physiology) and applied (i.e., bioprocesses) point of view. Pseudomonas putida KT2440 is a nonpathogenic soil bacterium endowed with the ability to adapt to a large variety of physicochemical and nutritional niches (Benedetti et al., 2016)(Nikel et al., 2014), and able to form biofilms depending on the environmental conditions (Volke and Nikel, 2018). Biofilm formation is under control of a complex gene regulatory network (GRN) that orchestrates the phenotypic switching mechanism (Huang et al., 2019)(Thomas et al., 2014). Due to the complexity of the GRN driving 
biofilm formation in P. putida, it is difficult to rely on a robust, single-cell proxy to characterize

2 the phenotypic switching involved in the process.

3 Propidium iodide (PI) is widely used for bacterial viability staining; it can only cross compromised bacterial membranes and is therefore considered an indicator of membrane integrity (Shi et al., 2007). However, recent studies demonstrated that PI might provide false-dead signals entering viable cells in some bacteria. Rosenberg et al. reported that PI staining is not indicative of membrane integrity in some instances, but rather binds extracellular DNA (eDNA), which is one of the components of bacterial extracellular polymeric substances (EPS) (Rosenberg et al., 2019). In most cases, the biofilm matrix represents around $90 \%$ of the total biofilm biomass and is mainly composed of polysaccharides, lipids, proteins, and eDNA (Fulaz et al., 2019). The contribution of eDNA for the biofilm's three-dimensional structure varies significantly among species (Beitelshees et al., 2018). Over the past few years, a great deal of attention was given to eDNA as one of the essential components of Pseudomonas sp.'s biofilm. In Pseudomonas aeruginosa, eDNA was found to represent a main component of biofilms. eDNA has also been reported to significantly affect the structural integrity of the biofilm matrix and protect bacterial cells in biofilms from physical stress, antibiotics, and detergents (Fulaz et al., 2019). Moreover, eDNA can be used as a source of nutrients for living cells and spread genetic material within the biofilm as well as planktonic populations (Chimileski et al., 2014)(Brown et al., 2015). In fact, eDNA plays a critical role in several steps of biofilm formation, including early bacterial attachment, microcolony formation and aggregation, and in determining the overall biofilm architecture (Qin et al., 2007)(Das et al., 2010)(Whitchurch et al., 2002). Recent reports suggest that the release of eDNA triggers bacterial surface adhesion by changing electrostatic and hydrophobic interactions between the bacterium and the surface and promotes early bacterial attachment and aggregation (Das et al., 2010)(Das et al., 2011)(Liu et al., 2008). Another study suggests that differences in the concentration of eDNA between Gram-negative (e.g. Pseudomonas aeruginosa PA14, Aeromonas hydrophila, and Escherichia coli) and Gram-positive species (e.g. Staphylococcus aureus, Staphylococcus epidermidis, and Enterococcus faecalis) explains the variations in aggregation and biofilm formation behavior (Das et al., 2014). Gram-negative bacterial strains displayed significant drops in aggregation when eDNA was removed through DNase I treatment (Das et al., 2014). 
2 In this work, the unique characteristics of eDNA was targeted in order to monitor biofilm switching

3 dynamics at a single cell level. In particular, by using PI staining, we validated the importance of

4 eDNA through different population states (i.e., planktonic phase, aggregation, and biofilm) and its

5 effect on phenotypic diversification. We demonstrate that early biofilm development can be

6 detected in planktonic cultures according to the degree of aggregation. Moreover, we demonstrate

7 that PI staining is not associated with modifications in the composition of the OM but depends on

8 the release of eDNA. The present study provides information on biofilm formation in P. putida

9 KT2440 with both a biofilm-defective mutant $(\triangle l a p A)$ and biofilm over-producing derivative

10 (overexpressing a mutated, hyperactive diguanylate cyclase), as well as in a natural isolate i.e.,

11 Pseudomonas composti. By applying a simple staining procedure relying on the use of PI, we were

12 able to track phenotypic heterogeneity during the switch from planktonic to sessile lifestyle.

13 Furthermore, we demonstrated that this property can be applied to identify and track biofilm-related

14 phenotypic switching in a continuous culture system.

\section{Results and discussion} switching related to biofilm formation

In order to investigate the link between a PI-positive subpopulation and eDNA, we performed a systematic analysis based on flow cytometry (FC) and confocal laser scanning microscopy (CLSM). Firstly, we conducted a 24-h batch phase mini-bioreactor cultivation of P. putida. Biofilm samples were collected at the end of the fermentation and treated with DNase I to remove eDNA; the untreated sample was used as control. The samples were stained with PI and analyzed by FC. In the FC analysis, the microbial population was divided into two subpopulations, according to PI uptake (PI-positive and PI negative). The PI-negative subpopulation had fluorescence values of around $10^{3}$ arbitrary units. These cells were considered to be unstained and thus having an intact, 
1 fluorescence values around $10^{5}$ arbitrary units was classified as PI-positive. Flow cytometry 2 analysis showed a significant difference in the PI-positive sub-population before and after treatment with DNase. The R1 region identified on the FC profiles corresponds to non-stained cells that were used as a negative control (Figure 1A). The majority of microbial cells were located in the $\mathrm{R} 1$ region before treated by the DNase enzyme, implied PI can bind with most of them (62.6\%) (Fig. 1B). In contrast, PI-positive sub-populations in biofilm decrease remarkably to $32 \%$ after treating with DNase, which could indicate that cells lose the capacity to bind PI after removing eDNA (Figure 1C).

9 To further verify the effect of eDNA on PI-positive sub-population, we performed confocal laser microscopy imaging on planktonic, aggregation, and biofilm samples with double straining (e.g., PI and SYTO9, TOTO-1 and SYTO60). Double-stained planktonic cells with PI and SYTO9 showed they were generally co-localized. We noticed green fluorescing cores under red-stained shells of individual sections and confirmed PI staining not being indicative of membrane integrity but rather staining of eDNA, which is present outside of intact membrane (Figure 2A and 2B), except for the most strongly red cells that lacked green signal and were apparently true dead signals. We visualized the percent of eDNA on aggregation and biofilm samples by combining SYTO60 and TOTO-1 using CLSM to produce high-quality images of eDNA. SYTO60 stains bacterial cell red, whereas TOTO-1 stains eDNA green (Figure 2C and 2D). We observed a high amount of eDNA in both samples tested. Moreover, to further prove the role of eDNA in PI-staining, we visualized DNase treated biofilm samples with CLSM. The results show that PI signals in a nontreated sample with DNase enzyme are intensively high compare to a sample that has been treated with DNase (Figure 2E and 2F).

Next, we investigated a possible correlation between eDNA and iron concentration and PI-positive subpopulation in Pseudomonas sp. Indeed, iron has been shown to play a critical role in altering the eDNA production and biofilm formation in diverse bacteria (Yang et al., 2007)(Allesen-Holm et al., 2006)(Banin et al., 2006)(Binnenkade et al., 2014)(Oh et al., 2018).

We have designed a matrix of iron concentrations gradient in a 96-well plate format. The extent of biofilm formation and quantity of eDNA has been assessed by crystal violet (CV) staining and fluorescent dye-based quantification, respectively. We found that biofilm formation of 
Pseudomonas decreased with increasing iron concentrations (Figure 3A), which is in accordance with the previous study (Yang et al., 2007). Moreover, the quantity of eDNA was assessed by using PicoGreen. The fluorescence intensity was measured immediately after adding the stain. Results demonstrated that the quantity of eDNA increased by decreasing iron concentration (Figure 3B).

In order to verify that the PI staining results are the direct consequence of biofilm formation, we genetically altered $P$. putida to either decrease or increase biofilm formation. To this end, we knocked out lapA, coding for the initial attachment protein. This strain, in the following called $\triangle l a p A$, is unable to attach to abiotic surfaces. Therefore, biofilm formation is severely reduced (Boyd et al., 2014). In order to increase biofilm formation, we transformed P. putida with the plasmid pS638::DGC-244, creating strain DGC. The introduced hyperactive di-guanylate cyclase increases the concentration of the second messenger cyclic-di-GMP (Christen et al., 2006). The concentration of this second messenger is a major regulator for biofilm formation i.e., high c-diGMP concentrations favor an adhesive lifestyle, while a low c-di-GMP concentration leads to a planktonic lifestyle (Gjermansen et al., 2006). As the basal expression of the di-guanylate cyclase led to a substantial increase in biofilm development, we refrained from supplementing the inducer to minimize the perturbation of the cells.

In addition, the percentage of PI positive-subpopulation in the presence of high $(100 \mu \mathrm{M})$ and low $(5 \mu \mathrm{M})$ iron $\left(\mathrm{Fe}^{3+}\right)$ concentration was investigated by $\mathrm{FC}$ in all four Pseudomonas strains. We have noticed in all tested strains, except $P$. putida $\triangle l a p A$, PI-positive subpopulation and biofilm eDNA with the biofilm matrix, we assumed a significant difference in the percentage of PI-positive subpopulation in the presence of high and low iron concentration could be due to a change in the quantity of eDNA present in the sample. For P. putida $\Delta l a p A$, as a non-biofilm former strain, we did not notice any change in PI- subpopulation or biofilm formation ability.

Considering the above-mentioned outcome, we evaluated the link between the biofilm-forming capacity and PI-positive sub-population by monitoring dynamic changes of PI-positive subpopulation during biofilm development. Results show a significant difference in PI-positive subpopulation among all tested strains. We observed high PI-positive percentage in DGC and $P$. 
composti by comparison with $P$. putida wild-type and the $\triangle l a p A$ derivative (Figure 4A). In addition, the PI-positive fraction in all cases was increased upon mid-exponential growth phase and decreased at the end of the batch phase (Figure 4A), which could be due to the release of eDNA and in the subsequent increase of aggregation at the beginning of the batch phase and decrease the amount of small aggregate in planktonic phase by switching to biofilm phase at the end of a phase (Figure S2). Meanwhile, we have evaluated the biofilm formation ability of all four strains using a crystal violet microtiter plate assay (Figure 4B). We have shown that DGC, and P. composti are strong biofilm former and produce accordingly more eDNA than the other strains tested.

Hereafter, due to the strong biofilm formation capability of DGC strain, planktonic cells, aggregates, and biofilm samples of this strain have been collected during the batch phase, and corresponding PI-positive fraction of samples have been compared. FC analysis indicates a higher PI-positive percentage (41.8\% and 30.8\%) in biofilm and aggregation samples, respectively, by comparison with the planktonic phase (7.5\%) (Figure 4C).

\section{OM permeability and porin composition are not implied in phenotypic switching related to} biofilm formation

Previous experiments have pointed out that PI binding could be related to a change in outer membrane (OM) composition (Sassi et al., 2019). Indeed, it has been shown that particular porin deletion in E. coli can lead to increased OM permeability according to PI staining (Brognaux et al., 2014) (Delvigne et al., 2011)(Brognaux et al., 2013). A systematic analysis of the effects of the group B porins on the outer membrane permeability of $P$. putida was then performed. Our aim was to investigate whether PI staining could also be related to modifying the porin composition in Pseudomonas OM. To this end, we constructed the single and multiple deletion mutants for all genes encoding OprB porins (OprB-I, OprB-II, and OprB-III) and measured the corresponding PIpositive subpopulation fraction through various time point starting from the late stationary phase. The proportion of PI-stained cells in the early stationary phase was low (0.2\%-2\%, data not shown). We have noticed that only 1-5\% subpopulation of cells was stained with PI across all time points, and that they exhibited the red fluorescence in FC in all of the tested mutants (Figure 5). This result 
indicates that single, double, and even triple knockouts of the $\operatorname{opr} B$ genes have no significant effect on the PI-positive subpopulation and that PI staining is not related to increased OM permeability

3 for P. putida. biofilm formation

To investigate the impact of pre-formed aggregates in the planktonic phase on early biofilm formation, we have monitored aggregation development during the batch phase cultivation for strains under study. The auto-aggregation properties of four strains $P$. putida, P. putida $\Delta l a p A, P$. putida DGC, and $P$. composti were measured over $24 \mathrm{~h}$ based on their sedimentation characteristics (Figure S3). Our results identified three auto-aggregation types, which have defined as follows. Strongly auto-aggregation $\left(\mathrm{Agg}^{+}\right)$strain $(\mathrm{DGC})$ showed significantly $(\mathrm{P}<0.01)$ higher autoaggregation percentages $(\geq 90 \%)$ and cells clumped together immediately, forming a precipitate and resulting in a clear solution; non-auto-aggregation $\left(\mathrm{Agg}^{-}\right)$strain $(\Delta l a p A)$, which was unable to auto-aggregate (auto-aggregation percentage <21\%) and produced consistent turbidity; and moderate Agg $\times$ strains ( $P$. putida and $P$. composti) showing auto-aggregation percentages between $20 \%$ to $70 \%$, their suspension showing both a precipitate and constant turbidity (Table S1). The result indicates that the biofilm-forming capability of strains under this study is highly related to auto-aggregation ability. The strong auto-aggregation strain DGC produces significantly higher biofilm compare to other tested strains.

On the other hand, in the same strains, aggregation in the planktonic phase was much more noticeable than for the two additional strains, which probably results in higher eDNA in the planktonic phase. In addition, to study the dynamic of aggregation formation during the batch phase, samples have been taken every $2 \mathrm{~h}$ and analyzed by FC. We have gated aggregation from the planktonic phase based on FSC and SSC signals. Flow cytometry analysis indicated the presence of aggregates phenotypic particles with a wide forward scatter distribution, indicative of variable particle sizes. These were distinguishable from single cells by their significantly bigger side- and forward- scatter values (Figure 6A). The degree of aggregation was measured for $P$. 
putida, $P$. putida $\triangle l a p A$, and $P$. composti during the batch phase (Figure 6B). However, in the case

2 of the DGC strain, the size of the aggregates was too high to be detected by FC. The aggregation

3 percentage was significantly higher in the natural isolate $P$. composti by comparison with $P$. putida and $P$. putida $\Delta l a p A$, which were also producing less biofilm. In $P$. putida and $P$. composti, we have noticed an increase of aggregation at the beginning of exponential growth, followed by a decrease upon entry into stationary phase together with the appearance of biofilm on the wall of the bioreactor. However, this phenomenon is hardly observed for P. putida $\Delta l a p$, in accordance with a very low cell aggregation.

Monitoring the phenotypic switching dynamics of Pseudomonas based on online flow cytometry allow for investigating biofilm lifecycle at a high temporal resolution

As indicated in the previous sections, PI has been identified as a useful biomarker to follow phenotypic switching from the planktonic phase to sessile life of $P$. putida. Accordingly, automated FC was then used for tracking this process during continuous culture with feast-to-famine cycles. This type of nutrient cycle is known to stimulate c-di-GMP synthesis and alter biofilm formation (Kalia et al., 2013)(Monds et al., 2007)(Gjermansen et al., 2010)(Gjermansen et al., 2005). The result pointed out that most of the cells are in a planktonic state during the batch phase (first $5 \mathrm{~h}$ ), and few cells are in aggregation $(<7 \%)$. Expectedly, the percentage of the corresponding PIpositive subpopulation was low $(<5 \%)$, suggesting reduced eDNA release at this stage. Besides these observations, a significant increase of the PI-positive subpopulation was observed after several feast to famine cycles. A gradual formation of a dense biofilm layer on the bioreactor wall was observed accordingly (Figure 7A and 7C). Intriguingly, the FC results show that the percentage of aggregation in the planktonic phase is fluctuating i.e., it rises during the first $20 \mathrm{~h}$ of continuous culture and then decreases. This non-monotonic behavior can be attributed to the accumulation of cells in the biofilm matrix during the first phase, making them impossible to detect by online FC. During the second stage, cells are released from the biofilm and can again be detected by FC (Figure 7B). The biofilm lifecycle can then be quantified at a very high time resolution based on this experimental set-up. 


\section{Discussion}

$4 \quad$ Studies about phenotypic diversification of microbial populations attract a lot of attention because of the possible new functionalities exhibiting diversified populations. A switch between planktonic and sessile growth is believed to result in a phenotypic change in bacteria. Several bacterial species benefit from phenotypic switching, which is used to generate population diversity, increase bacterial fitness, and adapt to fluctuating environments (Sousa et al., 2011). Biofilm development in P. putida undergoes various structural and metabolic changes that are tightly controlled through a complex GRN, including phenotypic switch. The current state of knowledge in biofilm development in P. putida has been limited to the systematic use of confocal microscopy on $P$. putida biofilms grown in flow cells or various strategies to classify and characterize genes involved in each stage of biofilm development (Fazli et al., 2014)(Klausen et $a l ., 2006)$. Despite the growing understanding of the biofilm cycle's structural and mechanistic details, little is known of the phenotypic switch during biofilm development in P. putida. As a result of the complexity of the GRN governing biofilm formation in P. putida, it is relatively challenging to depend on a robust single cell proxy to describe the phenotypic switching that takes place in this process. In the present work, we have identified PI as a useful biomarker to follow the cells switching from the planktonic phase to sessile life of $P$. putida. Our result from FC and CSLM indicates PI can bind to the eDNA present in a particular growth phase. The presence of eDNA, which is actively released through delegated cells, has gained significant interest (Okshevsky et al., 2015). Recent studies showed PI could significantly overestimate dead cell counts in the presence of eDNA (Rosenberg et al., 2019). Gião and Keevil noticed that co-staining of Listeria monocytogenes biofilms in tap water with PI and SYTO9 gives a false red signal, which is not an indication of dead cells. Still, this phenomenon it is due to the presence of eDNA (Gião and Keevil, 2014). Gallo also noted a similar picture staining the biofilm cells of Salmonella typhimurium strain carrying the reporter $\mathrm{P}_{c s g B A}:: g f p$ with PI. They confirmed green cells were surrounded by a red corona, demonstrating that eDNA was localized around live cells (Gallo et al., 2015). CLSM results 
biofilm on glass wool. They indicated that nucleic acid was linked with the cell exterior, where PI

2 had free access (Vilain et al., 2009).

3 We have shown that modifying the amount of eDNA by considering different iron concentrations

4 directly affects PI-positive sub-population. Iron has been demonstrated to be essential in the

5 modulation of eDNA production, for instance, by triggering prophage induction in Shewanella

6 oneidensis (Binnenkade et al., 2014), mediating cell lysis during biofilm formation in

7 Streptococcus pneumoniae (Trappetti et al., 2011), or, in the case of $P$. aeruginosa, regulating

8 eDNA production in response to both the $p q s$ quorum-sensing system and changes in the external

9 iron concentration (Allesen-Holm et al., 2006)(Yang et al., 2007). Our findings are in line with

10 studies that showed how high concentrations of iron suppress P. aeruginosa biofilm formation in

11 both microtitre plate cultivations and flow-chamber systems (Musk et al., 2005). Simultaneously,

12 low iron concentrations were reported to be necessary for P. aeruginosa biofilm (microcolony)

13 formation in an artificial sputum medium (Sriramulu et al., 2005). In Campylobacter jejuni, iron

14 supplementation increased the accumulation of total reactive oxygen species (ROS) and the

15 production of eDNA and extracellular polysaccharides, which leads to stimulating biofilms

16 formation (Oh et al., 2018).

17 Additionally, we observed that PI-positive sub-populations increase by manipulating the biofilm

18 formation ability of Pseudomonas sp. In light of eDNA interfering with viability staining, we

19 assume that the higher presence of eDNA leads to higher PI-positive fractions in strains producing

20 more biofilm. We have also shown that the percentage of PI-positive fraction is more elevated in

21 biofilm and aggregation samples as compared to planktonic cells. Since the quantity of eDNA is

higher in the aggregation and biofilm sample, the observed difference could arise due to the binding between eDNA and PI.

In traditional bacterial biofilm development models, biofilm is generally assumed to originate from individual cells seeded on a surface. However, many biofilm-forming bacteria tend to aggregate in the planktonic phase; therefore, there is a chance that many natural and infective biofilms may be rising entirely or partially from pre-formed cell aggregates. Yet how these aggregates influence biofilm initiation and development is not clear. Given the affinity of many bacteria to aggregate 
2010)(Monier and Lindow, 2003)(Stoodley et al., 2002), it seems likely that natural biofilms are

2 often initiated from pre-formed aggregates. P. aeruginosa, upon growth in liquid culture, forms

3 large aggregates containing massively packed viable cells and eDNA(Schleheck et al., 2009). For marine biofilms, the increased adhesiveness and surface conditioning of planktonic multicellular aggregates have been shown to accelerate bacterial attachment to a surface in early biofilm initiation (Bar-Zeev et al., 2012) . Similarly, for $P$. aeruginosa, an increased affinity toward aggregation, probably an alternative for greater stickiness, has also been associated with increased biofilm formation (Häußler et al., 2003)(Déziel et al., 2001). We have shown that it is possible to detect early biofilm formation in the planktonic phase by determining the degree of aggregation by FC. Our result shows auto-aggregation ability of Pseudomonas sp. was highly correlated to their biofilm formation ability.

Previous observations made in E. coli by using mutants of the KEIO library have been confirmed that porin suppression increases the occurrence of a partially PI-stained phenotype (Sassi et al., 2019). Although the mechanisms behind this increase in OM permeability are unknown, modifications in outer membrane porins composition have been hypothesized since porin removal has been shown to up and down-regulate other porins expression (Yang et al., 2011). Recently, it has been demonstrated that $E$. coli is capable of modifying the porin composition to adapt its OM permeability under nutrient limitation. Thus, E. coli differentiates into two subpopulations displaying individual phenotypic features (non-permeabilized and permeabilized cells) during glucose limitation (Sassi et al., 2019). In the case of $P$. putida, porins organization and regulation have been far less characterized. When compared with E. coli, the porins of strain KT2440 are organized in different gene and functional clusters, including membrane stabilization, transport of specific substrates, cell structure determination, and pore formation (Hancock and Brinkman, 2002). Among them, OprB has been more thoroughly explored and shows high homology with OprB from P. aeruginosa, which has been suggested to be involved in glucose uptake (Raneri et al., 2018). However, our results have shown that PI staining in Pseudomonas sp. is not associated with the modification of OM composition but is more likely depending on the release of eDNA.

It is known that nutrient conditions affect bacterial populations' transition between planktonic state 
1 al., 2012)(Paananen et al., 2003)(Gjermansen et al., 2010). In this work, we have used automated

2 FC for tracking the dynamics of the PI-positive subpopulation of $P$. putida upon exposure to

3 successive feast-to-famine cycles. Such cycling conditions promoted phenotypic switching

4 between planktonic cells, aggregates, and biofilm cells. The dynamic data acquired in this work

5 allows for tracking phenotypic switching based on PI staining, taking to account switching to a

6 stress condition effect the release of eDNA and biofilm formation (Ibáñez de Aldecoa et al.,

7 2017)(Lysis, 2018). Increasing the PI-positive sub-population also increased biofilm cells

8 appearance on the wall of bioreactor. FC results show online profiling of aggregates during

9 cultivation time, which helps us to monitor the phenotypic switch better. Taken together, the data

10 gathered in this work point out that the combination of online FC, continuous cultivation system

11 and appropriate single-cell proxy provides the user with the overall snapshot of phenotypic change

12 during the biofilm formation process. Further investigation would be required in order to fully

13 understand the mechanisms behind this phenotypic switch, for which our present method can be

14 further expanded and used to this end.

16 Conclusion

In the present study, PI was shown to be able bind with the viable cells from different phenotypes of Pseudomonas sp. We have demonstrated that the ubiquity of eDNA made it an ideal target for monitoring phenotypic switching of Pseudomonas in the biofilm development process by a simple staining procedure with PI. Hence, this study opens novel avenues to possible applications of PI as a single-cell proxy allowing us to capture the "key" subpopulation of cells involved in biofilm formation. In this sense, this work paves the way to further understand the phenotypic diversification mechanism involved in biofilm formation. This body of knowledge can be exploited to control phenotypic switch during transition between planktonic, aggregates, and biofilm states and subsequently control biofilm formation, e.g., in bioprocesses relying on 'catalytic' biofilms.

\section{Methods}




\section{Strains and medium composition}

2 Bacterial strains used in this study are listed in Table 1. All strains were maintained in 25\% (v/v)

3 glycerol at $-80^{\circ} \mathrm{C}$ in working seed vials $(2 \mathrm{~mL})$. Prior to experiments, one colony of each bacterium

4 was used to inoculate $10 \mathrm{~mL}$ of lysogeny broth (LB) medium $\left(10 \mathrm{~g} \mathrm{~L}^{-1} \mathrm{NaCl}, 5 \mathrm{~g} \mathrm{~L}^{-1}\right.$ yeast extract,

5 and $12 \mathrm{~g} \mathrm{~L}^{-1}$ tryptone) and grown for $6 \mathrm{~h}$ with shaking at $30^{\circ} \mathrm{C}$. Precultures and cultures of all bacteria were done in M9 minimal medium (33.7 mM Na $2 \mathrm{HPO}_{4}, 22.0 \mathrm{mM} \mathrm{KH} \mathrm{PO}_{4}, 8.55 \mathrm{mM}$ $\mathrm{NaCl}, 9.35 \mathrm{mM} \mathrm{NH}_{4} \mathrm{Cl}, 1 \mathrm{mM} \mathrm{MgSO}$, and $0.3 \mathrm{mM} \mathrm{CaCl}_{2}$ ), complemented with a trace elements (13.4 mM EDTA, $3.1 \mathrm{mM} \mathrm{FeCl}_{3} \cdot 6 \mathrm{H}_{2} \mathrm{O}, 0.62 \mathrm{mM} \mathrm{ZnCl}, 76 \mu \mathrm{M} \mathrm{CuCl}_{2} \cdot 2 \mathrm{H}_{2} \mathrm{O}, 42 \mu \mathrm{M} \mathrm{CoCl}_{2} \cdot 2 \mathrm{H}_{2} \mathrm{O}$, $162 \mu \mathrm{M} \mathrm{H}_{3} \mathrm{BO}_{3}$, and $\left.8.1 \mu \mathrm{M} \mathrm{MnCl}_{2} \cdot 4 \mathrm{H}_{2} \mathrm{O}\right), 1 \mu \mathrm{g} \mathrm{\textrm {L } ^ { - 1 }}$ biotin and $1 \mu \mathrm{g} \mathrm{\textrm {L } ^ { - 1 }}$ thiamin) and supplemented with glucose $\left(5 \mathrm{~g} \mathrm{~L}^{-1}\right)$ as the main carbon source $(\mathrm{pH}=7.2)$. For iron-dependent experiments, a concentrated $\mathrm{FeCl}_{3}$ solution was added to the media to give the final ferric iron concentrations described in the text. For strain DGC, the media was supplemented with gentamycin at a final 13 concentration of $10 \mu \mathrm{g} \mathrm{ml}^{-1}$.

\section{Plasmid construction and genetic manipulations}

Plasmid pS638::DGC was constructed by amplifying the hyperactive diguanylate cyclase mutant A0244 from Caulobacter crescentus (Christen et al., 2006), with the primer pair P1 and P2. The resulting amplicon and vector pSEVA638 were digested with BamHI and SacI (FD0054 and FD1133, Thermo Fisher Scientific). The fragments were purified (NucleoSpin Gel and PCR Clean-up Columns, Macherey Nagel) and ligated with T4 DNA ligase (EL0014, Thermo Fisher Scientific). E. coli DH5 $\alpha$ was transformed with the ligation mixture and the cell suspension was plated on a gentamycin-selective plate. Subsequently, the constructed pS638::DGC (where $D G C \mathrm{~A}^{0244}$ was placed under transcriptional control of the inducible $\mathrm{XylS} / \mathrm{Pm}$ expression system) plasmid was isolated from a single colony and its correctness was confirmed by sequencing. All the plasmids are listed in Table 2.

The plasmids for deletion of lapA and the porins oprB-I, oprB-II and oprB-III were constructed according to (Wirth et al., 2020b). In short, 500 bp homology arms (HA) flanking the gene coding sequences were amplified from chromosomal DNA of $P$. putida KT2440 using the primer pairs 

and assembled into the suicide vector pSNW2 employing the USER cloning method (Cavaleiro et $a l ., 2015)$. The purified plasmids were introduced into stationary $P$. putida KT2440 cells by electroporation and selected on LB agar medium supplemented with kanamycin $\left(50 \mu \mathrm{g} \mathrm{mL}^{-1}\right)$. The corresponding pSNW2 derivative, now fully integrated into the bacterial chromosome, was resolved by transforming the cells with the auxiliary plasmid pQURE6 and selection on gentamicin $\left(10 \mu \mathrm{g} \mathrm{mL}^{-1}\right)$ and 3-methylbenzoate $(1 \mathrm{mM})$ (Volke et al., 2020). Resolved strains (GFP-negative and kanamycin-sensitive) were tested for the desired genotype by colony PCR (OneTaq® $2 \mathrm{X}$ Master Mix with Standard Buffer, New England Biolabs).

\section{Mini-bioreactor cultivations}

A bioreactor system from $2 \mathrm{MAG} \odot$ block, equipped with eight positions for fermentation vessels of $16 \mathrm{~mL}$ total volume and a working volume of $12-\mathrm{mL}$ bioreactors, was used in these experiments. The bioreactor block is equipped with a magnetic inductive drive with two independent heat exchangers integrated into the bioreactor block, one for temperature control for the reaction mixture and the second to control the headspace's cooling of the bioreactors to prevent evaporation. The system is also equipped with fluorometric sensor spots for $\mathrm{pH}$ and dissolved oxygen (DO) positioned at the bottom of each reactor (MiniReaktor HTBD, Presens, Regensburg, Germany). We performed 24-h batch phase fermentations in order to test quickly and screen on PI-positive dynamic of strains under study, up to 6 replicates simultaneously. Samples have been taken every 1-2 $\mathrm{h}$ and immediately analyzed by FC.

\section{Sample preparation}

Adherent cells were harvested by scraping from the wall of the bioreactor vessel with a cell scraper and resuspended in PBS. In the case of aggregation, $5 \mathrm{~mL}$ culture was aliquoted into a $15-\mathrm{mL}$ Falcon tube, and aggregates were allowed to sediment for $10 \mathrm{~min}$. Then, aggregates were carefully picked up from the bottom of the tube by using sterile cut pipette tips, and transferred into $1.5-\mathrm{mL}$ 
Both aggregates and biofilm samples were resuspended in sterile PBS and vortexed for $30 \mathrm{~s}$. Before

2 FC analysis, samples were gently sonicated ( $2 \times$ for $12 \mathrm{~s}$, with $25 \%$ amplitude) to disperse clumps into single cells without causing cell lysis. A planktonic sample has been filtered through a sterile syringe filter $(5 \mu \mathrm{m})$ to eliminate aggregates before $\mathrm{FC}$ analysis.

\section{Bacteria staining protocols}

The four fluorescent stains used in this study were: (i) PI (P4170, Sigma-Aldrich); a stock solution was prepared at $1 \mathrm{mg} \mathrm{mL}^{-1}$ in sterile Milli-Q water and used at a final concentration of $1.5 \mu \mathrm{M}$ in sterilized PBS; (ii) TOTO ${ }^{\mathrm{TM}}-1$ iodide (T3600, Invitrogen ${ }^{\mathrm{TM}}$ Thermo Fisher Scientific); a stock solution was prepared in DMSO (at $1 \mathrm{mM}$ ) and used at a final concentration of $2 \mu \mathrm{M}$ in sterilized PBS; (iii) $\mathrm{SYTO}^{\mathrm{TM}}$ (S34854, Invitrogen ${ }^{\mathrm{TM}}$ Thermo Fisher Scientific); a stock solution was prepared in DMSO at $5 \mathrm{mM}$ and used at a final concentration of $5 \mu \mathrm{M}$ in sterilized PBS; and (iv) SYTO $^{\mathrm{TM}} 60$ (S11342, Invitrogen ${ }^{\mathrm{TM}}$ Thermo Fisher Scientific); a stock solution was prepared in DMSO at $5 \mathrm{mM}$ and used at a final concentration of $10 \mu \mathrm{M}$ in sterilized PBS. All bacterial samples were stained right before FC analysis by adding $1 \mu \mathrm{L}$ of the PI stock solution to $1 \mathrm{~mL}$ of a cell suspension in PBS $\left(1 \times 10^{7}\right.$ cells $\left.\mathrm{mL}^{-1}\right)$. The stained samples were incubated for $10 \mathrm{~min}$ in the dark at room temperature and analyzed by FC (live-dead gating was done based on heat-killed bacteria at $80^{\circ} \mathrm{C}$ for $\left.1 \mathrm{~h}\right)$.

\section{Flow cytometry}

FC analysis of PI-stained bacteria was carried out using BD Accuri ${ }^{\mathrm{TM}}$ C6 device (BD Biosciences).

Cell counts, red fluorescence (FL3), and Forward scatter (FSC) values were determined with FC. The software settings were as following: Fluidics, medium; Threshold, 20,000 on FSC-H; Run with limits, 10,000 events. Gating of dead and alive signal populations was executed on Propidium iodide (FL3-A; $670 \mathrm{~nm} \mathrm{LP)} \mathrm{scatter} \mathrm{plot.} \mathrm{FC} \mathrm{analysis} \mathrm{of} \mathrm{PI} \mathrm{staining} \mathrm{bacteria} \mathrm{was} \mathrm{carried} \mathrm{out} \mathrm{on}$ planktonic, aggregation, and biofilm samples. Cells were diluted to an appropriate density with filtered 1x phosphate-buffered saline (1× PBS; $8 \mathrm{~g} \mathrm{~L}^{-1} \mathrm{NaCl}, 0.2 \mathrm{~g} \mathrm{~L}^{-1} \mathrm{KCl}, 1.4 \mathrm{~g} \mathrm{~L}^{-1} \mathrm{Na}_{2} \mathrm{HPO}_{4}$, and $0.2 \mathrm{~g} \mathrm{~L}^{-1} \mathrm{KH}_{2} \mathrm{PO}_{4}$, pH 7.2) stained with PI and analyzed by flow cytometry. Online flow cytometry platform (Accuri C6 BD Biosciences San Jose CA USA) has been used in combination with a 
1 pulsed continuous cultivation system. The platform has been described in detail in a previous study

2 (Sassi et al., 2019). Briefly, sample processing following multiple steps, including sample

3 acquisition and online staining, dilution threshold, and online FC analysis. Flow cytometry data

4 were exported as an FCS file and processed by a custom Python script version (0.5.0).

\section{DNase enzyme Treatment}

Biofilm cells were prepared according to the protocol mentioned above. Then, the number of cells in the sample was adjusted to $\left(1 \times 10^{7}\right.$ cells $\left.\mathrm{mL}^{-1}\right)$, and samples were resuspended in $500 \mu \mathrm{L}$ of $1 \times$ DNase I buffer (10 mM Tris $\cdot \mathrm{HCl}, \mathrm{pH}=7.5,2.5 \mathrm{mM} \mathrm{MgCl}_{2}$, and $\left.0.1 \mathrm{mM} \mathrm{CaCl}\right)$ with or without DNase I (final concentration $160 \mathrm{U} \mathrm{mL}^{-1}$, Roche, cat. \# 04716728001) and were incubated at $37^{\circ} \mathrm{C}$ for $3 \mathrm{~h}$. After incubation, samples were pelleted by centrifugation at $8500 \mathrm{rpm}$ for $10 \mathrm{~min}$, resuspended in PBS, stained by PI, and analyzed by FCM.

All samples were analyzed by confocal laser-scanning microscope (LSM) LSM880 Airyscan super-resolution system (Carl Zeiss, Oberkochen, Germany). The images were taken with the PlanApochromat $63 \times / 1.4$ Oil objective. We used an excitation wavelength of $488 \mathrm{~nm}$ and emission at 500-550 nm for green fluorescence, and an excitation wavelength of $561 \mathrm{~nm}$ and emission at 580$615 \mathrm{~nm}$ for red fluorescence. Images were acquired continuously at a pixel resolution of $0.04 \mu \mathrm{m}$ (regular Airyscan mode) in XY and 1- $\mathrm{Xm}$ interval in $\mathrm{Z}$ step-size using the piezo drive. Before CLSM analysis, staining was performed with one or more fluorescent dyes in the following combinations: PI, SYTO9+PI, and TOTO1+SYTO60. An aliquot of 10-15 $\mu \mathrm{L}$ of 1:1 stain mixture solution has been added to a sample of planktonic, aggregate, and biofilm and incubated under exclusion of light (10 min for PI, SYTO9+PI, and $20 \mathrm{~min}$ for TOTO1+SYTO60).

\section{Auto-aggregation assay}

Auto-aggregation tests were adapted from the protocol published by Kos et al. (Kos et al., 2003).

For the auto-aggregation assay, the strains studied were first grown in M9 minimal medium for 18 
$1 \times \mathrm{PBS}$, and finally resuspended to $1 \times 10^{8} \mathrm{CFU} \mathrm{mL} \mathrm{m}^{-1}$ in the same medium. Then, suspensions were vortexed and incubated at room temperature for $15 \mathrm{~h}$. Samples were harvested during different times along the incubation experiment $(0,1,5,9$, and $15 \mathrm{~h})$. Each time, a $1-\mathrm{mL}$ aliquot from the top of the suspensions was carefully removed and its absorbance $(A)$ was read at $600 \mathrm{~nm}$ in a spectrophotometer. Auto-aggregation was calculated using the following formula:

$$
\text { Auto-aggregation }(\%)=\left[\left(A_{0}-A_{t}\right) / A_{0}\right] \times 100
$$

where $A_{0}$ indicates the absorbance at time $0 \mathrm{~h}$ and $A_{t}$ indicates the absorbance every hour, up to 15 h.

\section{Quantification of biofilm growth and eDNA in biofilms in 96-well plates}

Two-day biofilm formation of Pseudomonas strains was determined by crystal violet staining using a 96-well plate lid with pegs extending into each well (Nunc-TSP lid, Invitrogen ${ }^{\mathrm{TM}}$ Thermo Fisher Scientific). Briefly, precultures were grown overnight at $30^{\circ} \mathrm{C}$ to an $\mathrm{OD}_{600 \mathrm{~nm}}$ of 1.0. The cell suspensions were then adjusted to an $\mathrm{OD}_{600 \mathrm{~nm}}$ of 0.1 in M9 medium. A total of $160 \mu \mathrm{L}$ cell suspension were added to each well. Fresh medium was used as negative control. The plates were sealed with parafilm and incubated with shaking at $180 \mathrm{rpm}$ at $30^{\circ} \mathrm{C}$. The biofilm biomass was quantified with a crystal violet staining assay modified from previously reported CV assays (Ren et al., 2014). CV quantification was performed on the pegs of the Nunc-TSP lid culture system. Briefly, after $48 \mathrm{~h}$ of cultivation, the peg lids were taken out and washed three times using PBS. Subsequently, the peg lids were placed in plates with $180 \mu \mathrm{L}$ of an aqueous $1 \%(\mathrm{w} / \mathrm{v}) \mathrm{CV}$ solution. Then, the lids were washed with PBS three times after staining for 20 min. Subsequently, the peg lids with crystal violet stain were placed into a new microtiter plate with $200 \mu \mathrm{L}$ of $33 \%$ (w/v) glacial acetic acid in each well for $15 \mathrm{~min}$. The optical density at $590 \mathrm{~nm}$ of each sample was measured by a microplate reader (Tecan SPARK, Männedorf, Switzerland). The quantity of eDNA associated with Pseudomonas biofilm was examined in 96-well black plates (Cell culture microplate, $\left.\mu \mathrm{CLEAR}^{\circledR}\right)$ in triplicates parallel with the biofilm quantification. The 2-day biofilm culture was rinsed three times with sterile distilled water. eDNA was quantified by QuantiFluor 
1 dsDNA dye (QuantiFluor dsDNA System, Promega, Madison, WI, USA) according to

2 manufacturer's protocol. Briefly, eDNA in each sample was mixed with $200 \mu \mathrm{L}$ of freshly prepared

3 QuantiFluor dsDNA dye in TE buffer. Wells with QuantiFluor dsDNA dye were incubated for 5

4 min before measuring the fluorescence intensity (excitation wavelength $=504 \mathrm{~nm}$ and emission

5 wavelength $=531 \mathrm{~nm}$ ) using a Tecan microplate reader. Lambda DNA (Invitrogen ${ }^{\mathrm{TM}}$ Molecular

6 Probes) was used to generate a calibration curve for each run.

8 Pulsed continuous cultivation with online flow cytometry

$9 \quad$ P. putida KT2440 was cultivated in a stirred bioreactor (Biostat B-Twin, Sartorius) in continuous

10 mode with glucose feed pulses $\left(0.5 \mathrm{~g} \mathrm{~L}^{-1}\right.$ per pulse $)$ at a frequency of one pulse per hour. Data

11 acquisition was performed every 12 min via a coupled, online FC analysis. A 1-L volume of M9

12 minimal medium (containing $5 \mathrm{~g} \mathrm{~L}^{-1}$ glucose) was inoculated by overnight preculture $\left(\mathrm{OD}_{600}=0.3\right)$

13 in the 2-L capacity bioreactor. The $\mathrm{pH}$ and temperature were maintained at 7.2 and $30^{\circ} \mathrm{C}$,

14 respectively. Stirring was set at $800 \mathrm{rpm}$ and aeration flow rate to $1 \mathrm{~L} \mathrm{~min}^{-1}(1 \mathrm{vvm})$. The drop in

15 DO marked the depletion of oxygen and the start of continuous cultivation mode. M9 minimal

16 medium was fed without carbon source into the bioreactor at a dilution rate $D=0.1 \mathrm{~h}^{-1}$. After $1 \mathrm{~h}$,

17 a pulse of glucose was injected in order to increase the concentration as fast as possible (within 30

$18 \mathrm{~s})$ to a global glucose concentration of $0.5 \mathrm{~g} \mathrm{~L}^{-1}$. The microbial population was tracked by an online

19 flow cytometry device as previously described (Nguyen et al., 2021)(Sassi et al., 2019). Briefly, a

$20 \quad 0.8-\mathrm{mL}$ sample was taken from the bioreactor each $12 \mathrm{~min}$, automatically diluted and stained with

21 PI, and then automatically injected into an Accuri C6 FC for detection. Before processing, the independent .fcs files obtained through online FC were compacted in a dataframe (.pkl file extension) based on the Pandas package (https://pandas.pydata.org/) from Python. This file was used for generating the plots with the evolution of PI-positive and aggregates subpopulations, as well as for generating individual dotplots with forward scatter (FSC) as $x$-axis and PI fluorescence as $y$-axis. These dotplots were further assembled into a single avi movie file based on the ImageJ software (Rueden et al., 2017) shown as Supplemental material (Movies S1). Raw .fcs data were deposited on FlowRepository and can be accessed by following the link: 

wVN5sllGgBYFgsRyHp

\section{References}

Ackermann, M. (2015) A functional perspective on phenotypic heterogeneity in microorganisms. Nat Rev Microbiol 13: 497-508.

Allesen-Holm, M., Barken, K.B., Yang, L., Klausen, M., Webb, J.S., Kjelleberg, S., et al. (2006) A characterization of DNA release in Pseudomonas aeruginosa cultures and biofilms. $\mathrm{Mol}$ Microbiol 59: 1114-1128.

Bagdasarian, M., Lurz, R., Rückert, B., Franklin, F.C.H., Bagdasarian, M.M., Frey, J., and Timmis, K.N. (1981) Specific-purpose plasmid cloning vectors II. Broad host range, high copy number, RSF 1010-derived vectors, and a host-vector system for gene cloning in Pseudomonas. Gene 16: 237-247.

Banin, E., Brady, K.M., and Greenberg, E.P. (2006) Chelator-induced dispersal and killing of Pseudomonas aeruginosa cells in a biofilm. Appl Environ Microbiol 72: 2064-2069.

Bar-Zeev, E., Berman-Frank, I., Girshevitz, O., and Berman, T. (2012) Revised paradigm of aquatic biofilm formation facilitated by microgel transparent exopolymer particles. Proc Natl Acad Sci U S A 109: 9119-9124.

Beitelshees, M., Hill, A., Jones, C.H., and Pfeifer, B.A. (2018) Phenotypic variation during biofilm formation: Implications for anti-biofilm therapeutic design. Materials (Basel) 11: 118.

Benedetti, I., de Lorenzo, V., and Nikel, P.I. (2016) Genetic programming of catalytic Pseudomonas putida biofilms for boosting biodegradation of haloalkanes. Metab Eng 33: 109-118.

Berlanga, M. and Guerrero, R. (2016) Living together in biofilms: The microbial cell factory and its biotechnological implications. Microb Cell Fact 15: 1-11.

Binnenkade, L., Teichmann, L., and Thormanna, K.M. (2014) Iron triggers $\lambda$ So prophage induction and release of extracellular DNA in shewanella oneidensis MR-1 biofilms. Appl Environ Microbiol 80: 5304-5316.

Boyd, C.D., Jarrod Smith, T., El-Kirat-Chatel, S., Newell, P.D., Dufrêne, Y.F., and O'Toolea, G.A. (2014) Structural features of the Pseudomonas fluorescens biofilm adhesin LapA required for LapG-dependent cleavage, biofilm formation, and cell surface localization. $J$ Bacteriol 196: 2775-2788.

Brognaux, A., Bugge, J., Schwartz, F.H., Thonart, P., Telek, S., and Delvigne, F. (2013) Realtime monitoring of cell viability and cell density on the basis of a three dimensional optical reflectance method (3D-ORM): Investigation of the effect of sub-lethal and lethal injuries. $J$ Ind Microbiol Biotechnol 40: 679-686.

Brognaux, A., Francis, F., Twizere, J.C., Thonart, P., and Delvigne, F. (2014) Scale-down effect on the extracellular proteome of Escherichia coli: Correlation with membrane permeability and modulation according to substrate heterogeneities. Bioprocess Biosyst Eng 37: 14691485.

Brown, H.L., Reuter, M., Hanman, K., Betts, R.P., and Van Vliet, A.H.M. (2015) Prevention of biofilm formation and removal of existing biofilms by extracellular dnases of campylobacter jejuni. PLoS One 10: 1-21.

Burmølle, M., Thomsen, T.R., Fazli, M., Dige, I., Christensen, L., Homøe, P., et al. (2010) Biofilms in chronic infections - A matter of opportunity - Monospecies biofilms in multispecies infections. FEMS Immunol Med Microbiol 59: 324-336.

Cavaleiro, A.M., Kim, S.H., Seppälä, S., Nielsen, M.T., and Nørholm, M.H.H. (2015) Accurate DNA Assembly and Genome Engineering with Optimized Uracil Excision Cloning. ACS 
Synth Biol 4: 1042-1046.

Chimileski, S., Franklin, M.J., and Papke, R.T. (2014) Biofilms formed by the archaeon Haloferax volcanii exhibit cellular differentiation and social motility, and facilitate horizontal gene transfer. BMC Biol 12:.

Christen, B., Christen, M., Paul, R., Schmid, F., Folcher, M., Jenoe, P., et al. (2006) Allosteric Control of Cyclic di-GMP Signaling. J Biol Chem 281: 32015-32024.

Das, T., Krom, B.P., Van Der Mei, H.C., Busscher, H.J., and Sharma, P.K. (2011) DNAmediated bacterial aggregation is dictated by acid-base interactions. Soft Matter 7: 29272935.

Das, T., Sehar, S., Koop, L., Wong, Y.K., Ahmed, S., Siddiqui, K.S., and Manefield, M. (2014) Influence of calcium in extracellular DNA mediated bacterial aggregation and biofilm formation. PLoS One 9: 1-11.

Das, T., Sharma, P.K., Busscher, H.J., Van Der Mei, H.C., and Krom, B.P. (2010) Role of extracellular DNA in initial bacterial adhesion and surface aggregation. Appl Environ Microbiol 76: 3405-3408.

Delvigne, F., Brognaux, A., Francis, F., Twizere, J.C., Gorret, N., Sorensen, S.J., and Thonart, P. (2011) Green fluorescent protein (GFP) leakage from microbial biosensors provides useful information for the evaluation of the scale-down effect. Biotechnol J 6: 968-978.

Delvigne, F., Zune, Q., Lara, A.R., Al-Soud, W., and Sørensen, S.J. (2014) Metabolic variability in bioprocessing: Implications of microbial phenotypic heterogeneity. Trends Biotechnol 32: 608-616.

Déziel, E., Comeau, Y., and Villemur, R. (2001) Initiation of biofilm formation by Pseudomonas aeruginosa 57RP correlates with emergence of hyperpiliated and highly adherent phenotypic variants deficient in swimming, swarming, and twitching motilities. J Bacteriol 183: 11951204.

Drenkard, E. (2003) Antimicrobial resistance of Pseudomonas aeruginosa biofilms. Microbes Infect 5: 1213-1219.

Eldar, A. and Elowitz, M.B. (2010) Functional roles for noise in genetic circuits. Nature 467: 167-173.

Fazli, M., Almblad, H., Rybtke, M.L., Givskov, M., Eberl, L., and Tolker-Nielsen, T. (2014) Regulation of biofilm formation in Pseudomonas and Burkholderia species. Environ Microbiol 16: 1961-1981.

Fulaz, S., Vitale, S., Quinn, L., and Casey, E. (2019) Nanoparticle-Biofilm Interactions: The Role of the EPS Matrix. Trends Microbiol 27: 915-926.

Gallo, P.M., Rapsinski, G.J., Wilson, R.P., Oppong, G.O., Sriram, U., Goulian, M., et al. (2015) Amyloid-DNA Composites of Bacterial Biofilms Stimulate Autoimmunity. Immunity 42: 1171-1184.

Gião, M.S. and Keevil, C.W. (2014) Listeria monocytogenes can form biofilms in tap water and enter into the viable but non-cultivable state. Microb Ecol 67: 603-611.

Gjermansen, M., Nilsson, M., Yang, L., and Tolker-Nielsen, T. (2010) Characterization of starvation-induced dispersion in Pseudomonas putida biofilms: genetic elements and molecular mechanisms. Mol Microbiol 75: 815-826.

Gjermansen, M., Ragas, P., Sternberg, C., Molin, S., and Tolker-Nielsen, T. (2005) Characterization of starvation-induced dispersion in Pseudomonas putida biofilms. Environ Microbiol 7: 894-904.

Gjermansen, M., Ragas, P., and Tolker-Nielsen, T. (2006) Proteins with GGDEF and EAL domains regulate Pseudomonas putida biofilm formation and dispersal. FEMS Microbiol Lett 265: 215-224.

Hancock, R.E.W. and Brinkman, F.S.L. (2002) Function of Pseudomonas porins in uptake and efflux. Annu Rev Microbiol 56: 17-38.

Häußler, S., Ziegler, I., Löttel, A., Götz, F. V., Rohde, M., Wehmhöhner, D., et al. (2003) Highly adherent small-colony variants of Pseudomonas aeruginosa in cystic fibrosis lung infection. J Med Microbiol 52: 295-301.

Huang, M.Y., Woolford, C.A., May, G., Joel Mcmanus, C., and Mitchell, A.P. (2019) Circuit 
diversification in a biofilm regulatory network. PLoS Pathog 15: 1-26.

Ibáñez de Aldecoa, A.L., Zafra, O., and González-Pastor, J.E. (2017) Mechanisms and regulation of extracellular DNA release and its biological roles in microbial communities. Front Microbiol 8: 1-19.

Kalia, D., Merey, G., Nakayama, S., Zheng, Y., Zhou, J., Luo, Y., et al. (2013) Nucleotide, c-diGMP, c-di-AMP, cGMP, cAMP, (p)ppGpp signaling in bacteria and implications in pathogenesis. Chem Soc Rev 42: 305-341.

Klausen, M., Gjermansen, M., Kreft, J.U., and Tolker-Nielsen, T. (2006) Dynamics of development and dispersal in sessile microbial communities: Examples from Pseudomonas aeruginosa and Pseudomonas putida model biofilms. FEMS Microbiol Lett 261: 1-11.

Kos, B., Sušković, J., Vuković, S., Šmpraga, M., Frece, J., and Matošić, S. (2003) Adhesion and aggregation ability of probiotic strain Lactobacillus acidophilus M92. J Appl Microbiol 94: 981-987.

Liu, H.H., Yang, Y.R., Shen, X.C., Zhang, Z.L., Shen, P., and Xie, Z.X. (2008) Role of DNA in bacterial aggregation. Curr Microbiol 57: 139-144.

Lysis, E.S.B. (2018) crossm. 84: 1-18.

Mah, T.F.C. and O'Toole, G.A. (2001) Mechanisms of biofilm resistance to antimicrobial agents. Trends Microbiol 9: 34-39.

Martínez-Garćía, E., Aparicio, T., Goñi-Moreno, A., Fraile, S., and De Lorenzo, V. (2015) SEVA 2.0: An update of the Standard European Vector Architecture for de-/re-construction of bacterial functionalities. Nucleic Acids Res 43: D1183-D1189.

McDougald, D., Rice, S.A., Barraud, N., Steinberg, P.D., and Kjelleberg, S. (2012) Should we stay or should we go: Mechanisms and ecological consequences for biofilm dispersal. Nat Rev Microbiol 10: 39-50.

Monds, R.D., Newell, P.D., Gross, R.H., and O'Toole, G.A. (2007) Phosphate-dependent modulation of c-di-GMP levels regulates Pseudomonas fluorescens Pf0-1 biofilm formation by controlling secretion of the adhesin LapA. Mol Microbiol 63: 656-679.

Monier, J.M. and Lindow, S.E. (2003) Differential survival of solitary and aggregated bacterial cells promotes aggregate formation on leaf surfaces. Proc Natl Acad Sci U S A 100: 1597715982.

Musk, D.J., Banko, D.A., and Hergenrother, P.J. (2005) Iron salts perturb biofilm formation and disrupt existing biofilms of Pseudomonas aeruginosa. Chem Biol 12: 789-796.

Nguyen, T.M., Telek, S., Zicler, A., Andres Martinez, J., Zacchetti, B., Kopp, J., et al. (2021) Reducing phenotypic and genotypic instabilities of microbial population during continuous cultivation based on stochastic switching dynamics. bioRxiv 2021.01.13.426484.

Nikel, P.I., Martínez-García, E., and De Lorenzo, V. (2014) Biotechnological domestication of pseudomonads using synthetic biology. Nat Rev Microbiol 12: 368-379.

Oh, E., Andrews, K.J., and Jeon, B. (2018) Enhanced biofilm formation by ferrous and ferric iron through oxidative stress in Campylobacter jejuni. Front Microbiol 9: 1-9.

Okshevsky, M., Regina, V.R., and Meyer, R.L. (2015) Extracellular DNA as a target for biofilm control. Curr Opin Biotechnol 33: 73-80.

Paananen, A., Vuorimaa, E., Torkkeli, M., Penttilä, M., Kauranen, M., Ikkala, O., et al. (2003) Structural hierarchy in molecular films of two class II hydrophobins. Biochemistry $\mathbf{4 2}$ : 5253-8.

Platt, R., Drescher, C., Park, S.K., and Phillips, G.J. (2000) Genetic system for reversible integration of DNA constructs and lacZ gene fusions into the Escherichia coli chromosome. Plasmid 43: 12-23.

Qin, Z., Ou, Y., Yang, L., Zhu, Y., Tolker-Nielsen, T., Molin, S., and Qu, D. (2007) Role of autolysin-mediated DNA release in biofilm formation of Staphylococcus epidermidis. Microbiology 153: 2083-2092.

Raneri, M., Pinatel, E., Peano, C., Rampioni, G., Leoni, L., Bianconi, I., et al. (2018) Pseudomonas aeruginosa mutants defective in glucose uptake have pleiotropic phenotype and altered virulence in non-mammal infection models. Sci Rep 8: 1-15.

Ren, D., Madsen, J.S., de la Cruz-Perera, C.I., Bergmark, L., Sørensen, S.J., and Burmølle, M. 
(2014) High-Throughput Screening of Multispecies Biofilm Formation and Quantitative PCR-Based Assessment of Individual Species Proportions, Useful for Exploring Interspecific Bacterial Interactions. Microb Ecol 68: 146-154.

Rosenberg, M., Azevedo, N.F., and Ivask, A. (2019) Propidium iodide staining underestimates viability of adherent bacterial cells. Sci Rep 9: 1-14.

Rueden, C.T., Schindelin, J., Hiner, M.C., DeZonia, B.E., Walter, A.E., Arena, E.T., and Eliceiri, K.W. (2017) ImageJ2: ImageJ for the next generation of scientific image data. BMC Bioinformatics 18:.

Sassi, H., Nguyen, T.M., Telek, S., Gosset, G., Grünberger, A., and Delvigne, F. (2019) Segregostat: a novel concept to control phenotypic diversification dynamics on the example of Gram-negative bacteria. Microb Biotechnol 12: 1064-1075.

Schleheck, D., Barraud, N., Klebensberger, J., Webb, J.S., McDougald, D., Rice, S.A., and Kjelleberg, S. (2009) Pseudomonas aeruginosa PAO1 Preferentially Grows as Aggregates in Liquid Batch Cultures and Disperses upon Starvation. PLoS One 4: e5513.

Schreiber, F., Littmann, S., Lavik, G., Escrig, S., Meibom, A., Kuypers, M.M.M., and Ackermann, M. (2016) Phenotypic heterogeneity driven by nutrient limitation promotes growth in fluctuating environments. Nat Microbiol 1: 1-7.

Shi, L., Günther, S., Hübschmann, T., Wick, L.Y., Harms, H., and Müller, S. (2007) Limits of propidium iodide as a cell viability indicator for environmental bacteria. Cytom Part A 71: 592-598.

Sousa, A.M., Machado, I., and Pereira, M.O. (2011) Phenotypic switching: an opportunity to bacteria thrive. Sci against Microb Pathog Commun Curr Res Technol Adv 252-262.

Sriramulu, D.D., Lünsdorf, H., Lam, J.S., and Römling, U. (2005) Microcolony formation: A novel biofilm model of Pseudomonas aeruginosa for the cystic fibrosis lung. $J$ Med Microbiol 54: 667-676.

Stoodley, P., Sauer, K., Davies, D.G., and Costerton, J.W. (2002) Biofilms as complex differentiated communities. Annu Rev Microbiol 56: 187-209.

Thomas, P., Popovic, N., and Grima, R. (2014) Phenotypic switching in gene regulatory networks. Proc Natl Acad Sci U S A 111: 6994-6999.

Trappetti, C., Gualdi, L., Di Meola, L., Jain, P., Korir, C.C., Edmonds, P., et al. (2011) The impact of the competence quorum sensing system on Streptococcus pneumoniae biofilms varies depending on the experimental model. BMC Microbiol 11: 75.

Vilain, S., Pretorius, J.M., Theron, J., and Brözel, V.S. (2009) DNA as an adhesin: Bacillus cereus requires extracellular DNA to form biofilms. Appl Environ Microbiol 75: 2861-2868.

Volke, D.C., Friis, L., Wirth, N.T., Turlin, J., and Nikel, P.I. (2020) Synthetic control of plasmid replication enables target- and self-curing of vectors and expedites genome engineering of Pseudomonas putida. Metab Eng Commun 10: e00126.

Volke, D.C. and Nikel, P.I. (2018) Getting Bacteria in Shape: Synthetic Morphology Approaches for the Design of Efficient Microbial Cell Factories. Adv Biosyst 2: 1800111.

Whitchurch, C.B., Tolker-Nielsen, T., Ragas, P.C., and Mattick, J.S. (2002) Extracellular DNA required for bacterial biofilm formation. Science (80- ) 295: 1487.

Wirth, N.T., Kozaeva, E., and Nikel, P.I. (2020a) Accelerated genome engineering of Pseudomonas putida by I-SceI-mediated recombination and CRISPR-Cas9 counterselection. Microb Biotechnol 13: 233-249.

Wirth, N.T.,.

Worsey, M.J. and Williams, A.P. (1975) Metablism of toluene and xylenes by Pseudomonas putida (arvilla) mt 2: evidence for a new function of the TOL plasmid. J Bacteriol 124: 713.

Yang, J.N., Wang, C., Guo, C., Peng, X.X., and Li, H. (2011) Outer membrane proteome and its regulation networks in response to glucose concentration changes in Escherichia coli. $\mathrm{Mol}$ Biosyst 7: 3087-3093.

Yang, L., Barken, K.B., Skindersoe, M.E., Christensen, A.B., Givskov, M., and Tolker-Nielsen, T. (2007) Effects of iron on DNA release and biofilm development by Pseudomonas aeruginosa. Microbiology 153: 1318-1328. 
bioRxiv preprint doi: https://doi.org/10.1101/2021.02.11.430776; this version posted February 11, 2021. The copyright holder for this preprint (which was not certified by peer review) is the author/funder, who has granted bioRxiv a license to display the preprint in perpetuity. It is made available under aCC-BY-NC-ND 4.0 International license.

1 
1 Table 1. Bacterial strains and plasmids used in this study.

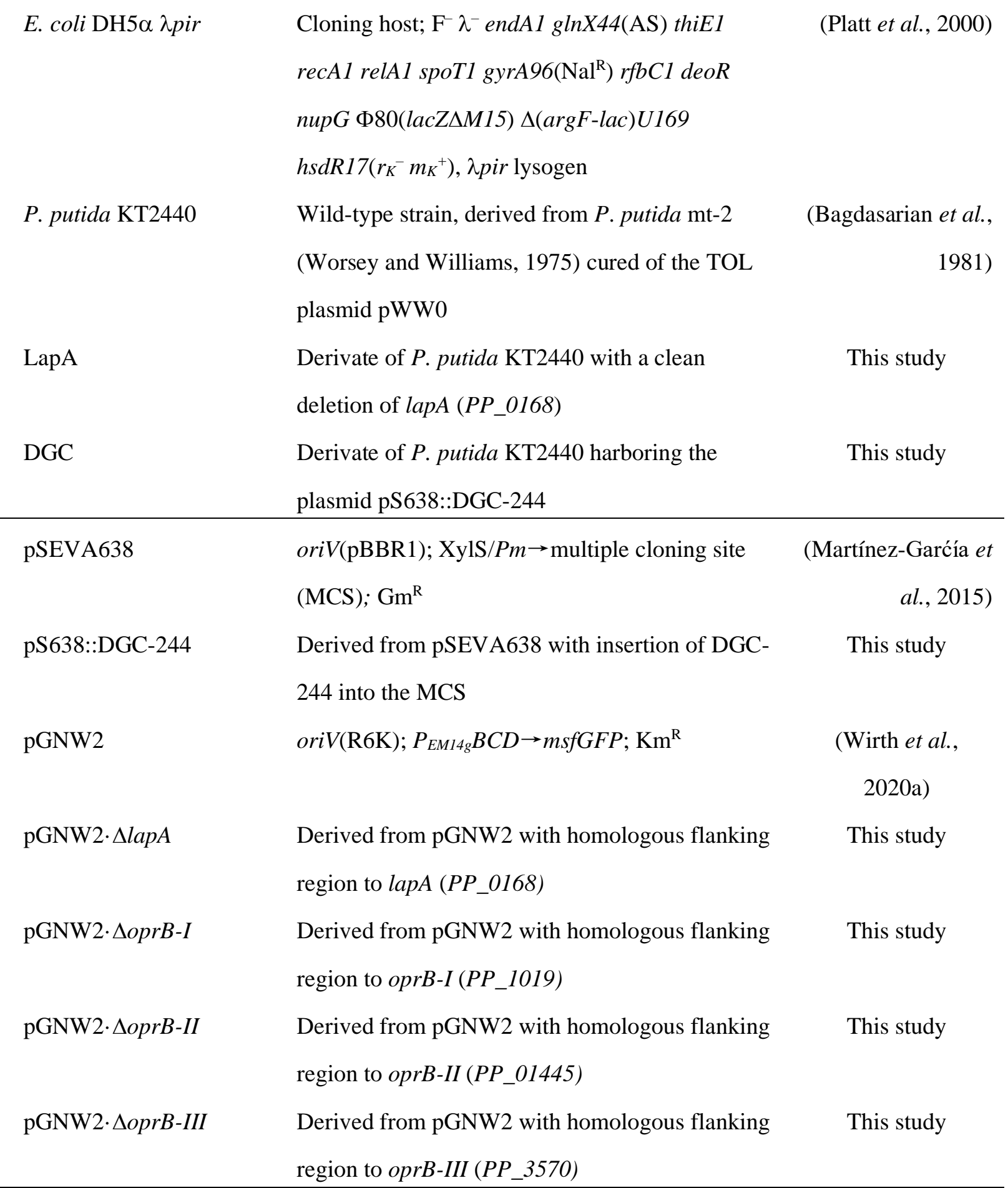


2 Table 2. Oligonucleotides used in this study

\begin{tabular}{|c|c|c|}
\hline Oligonucleotide & Sequence $\left(5^{\prime} \rightarrow 3^{\prime}\right)$ & Use \\
\hline $\mathrm{P} 1$ & AAA $G A G C T C$ TTA GGA GGA AAA ACA TAT GAA & \multirow{3}{*}{$\begin{array}{l}\text { Amplification of } \\
d g c \mathrm{~A} 0240\end{array}$} \\
\hline & AAT CTC AGG CGC CCG GAC & \\
\hline $\mathrm{P} 2$ & AAA GGA TCC TCA AGC GCT CCT GCG CTT G & \\
\hline $\mathrm{P} 3 \dagger$ & AGA TCC UAT TCA TCT ATA GAG TGC GGA TTC & \multirow{4}{*}{$\begin{array}{l}\text { Amplification of } \\
\text { genomic regions } \\
\text { adjacent to lapA }\end{array}$} \\
\hline $\mathrm{P} 4 \uparrow$ & ATT GGA CUC TCC GTGTGACCCGATGG & \\
\hline $\mathrm{P} 5 \dagger$ & AGT CCA AUG TGA CAG ACC ACC GGG GCC & \\
\hline $\mathrm{P} 6 \dagger$ & AGG TCG ACU TCG ATT GGT CGA CGG GTA CG & \\
\hline $\mathrm{P} 7 \dagger$ & AGA TCC UCC GCA GCA GAT CTA CAA CG & \multirow{5}{*}{$\begin{array}{l}\text { Amplification of } \\
\text { genomic regions } \\
\text { adjacent to oprB-I }\end{array}$} \\
\hline $\mathrm{P} 8 \dagger$ & ACA TCC CUT TGC GTC CTC TTT & \\
\hline $\mathrm{P} 9 \dagger$ & AGG GAT GUG AGG CAA CTT GTT GTA AAT TTA & \\
\hline & CG & \\
\hline $\mathrm{P} 10 \dagger$ & AGG TCG ACU GCT GTC CTG ATG TTC GGT G & \\
\hline $\mathrm{P} 11 \uparrow$ & AGA TCC UAA ACC CGC CAA CGA AAC C & \multirow{4}{*}{$\begin{array}{l}\text { Amplification of } \\
\text { genomic regions } \\
\text { adjacent to oprB-II }\end{array}$} \\
\hline $\mathrm{P} 12 \uparrow$ & ACA TGA GAU AGC GCT ATC TTT TGATT & \\
\hline $\mathrm{P} 13 \dagger$ & ATC TCA TGU AAC CCC TTT TTT GAC CTG ACG & \\
\hline $\mathrm{P} 14 \dagger$ & AGG TCG ACU GTA GGC CTG CCA TTC GC & \\
\hline $\mathrm{P} 15 \dagger$ & AGA TCC UAT GCC GTG AAC AAG AAC CG & \multirow{4}{*}{$\begin{array}{l}\text { Amplification of } \\
\text { genomic regions } \\
\text { adjacent to oprB-III }\end{array}$} \\
\hline $\mathrm{P} 16 \dagger$ & ATT ACA GAA UCT CGG GTT GTC TTT G & \\
\hline $\mathrm{P} 17 \dagger$ & ATT CTG TAA UGT GAA CCG CCG GGG CCG C & \\
\hline $\mathrm{P} 18 \dagger$ & AGG TCG ACU GCT TCG ATC CAC CGT TCT C & \\
\hline
\end{tabular}


A

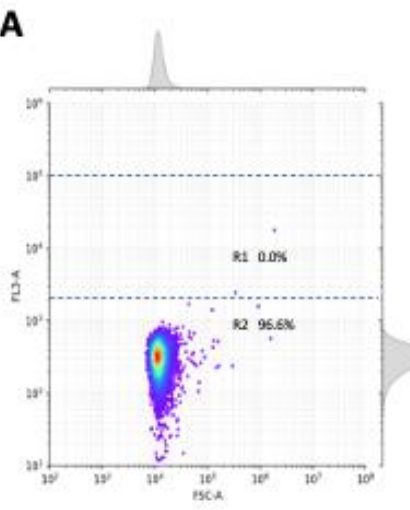

B

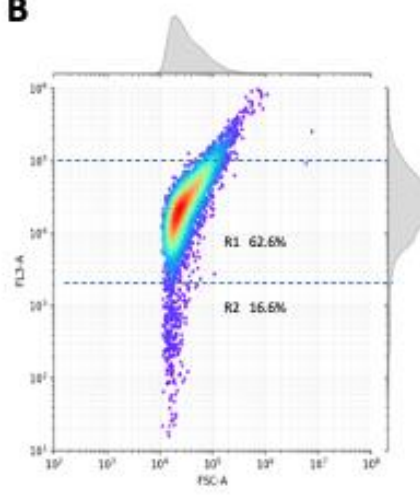

C

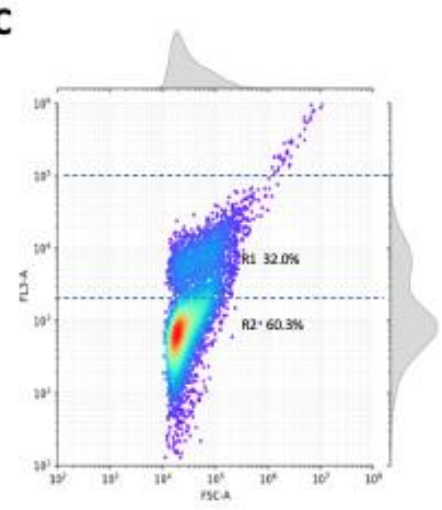

2 Figure 1. Flow cytometry analysis illustrating the effects of DNase treatment on PI-positive

3 fraction of biofilm sample. A Negative control shows non-stained cells according to R1 region on

4 the FC profiles. B Sample showing high PI uptake, before treated by the DNase enzyme, the

5 majority of microbial cells were located in the R1 region (62.6\%). C Sample After treating with

6 DNase enzyme, the PI-positive fraction decreases significantly (32.0\%), as a result of eDNA 7 hydrolysis. 

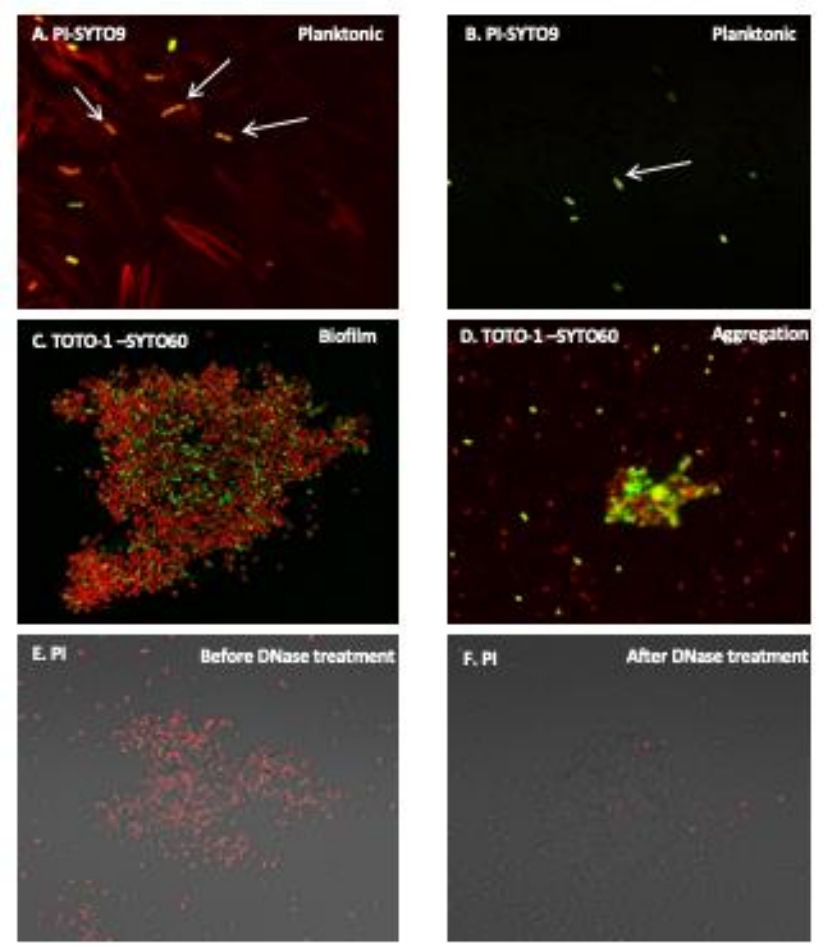

2 Figure 2. Confocal laser scanning microscopy (CSLM) images of P. putida KT2440 (planktonic, 3 aggregation and biofilm) samples. A-B Planktonic sample co-stained with propidium iodide (PI) 4 and SYTO9. C Biofilm cells (co-stained with TOTO-1 and SYTO60). D Cell aggregates stained 5 with a combination of TOTO-1 and SYTO60. E Biofilm cells stained with PI before treatment with 6 DNase enzyme. F Biofilm cells stained with PI after being treated with DNase enzyme. 
A
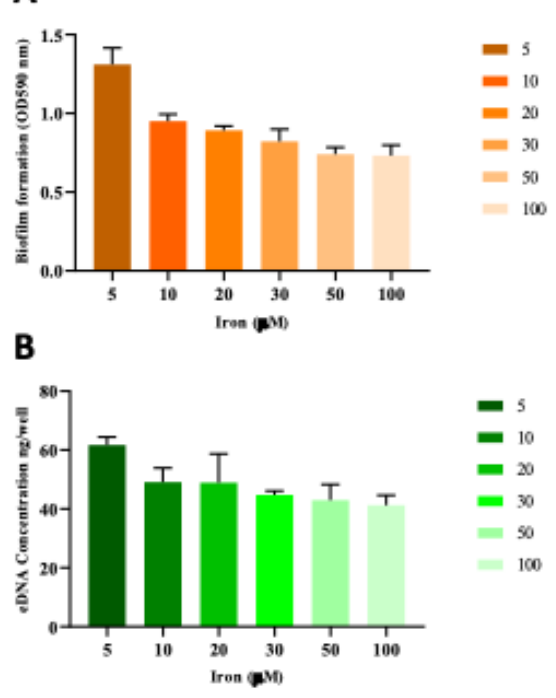

C

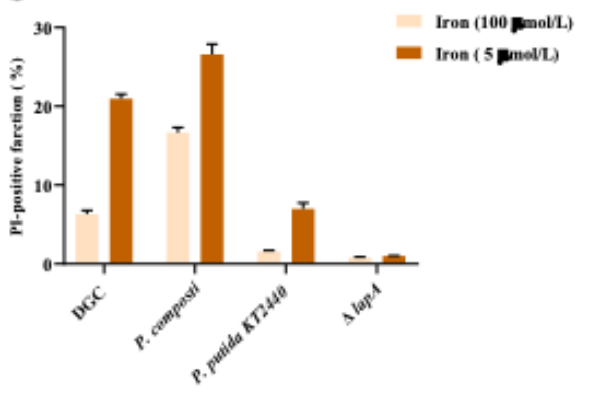

2 Figure 3. Effect of iron concentration on biofilm formation, extracellular DNA and the PI-positive 3 subpopulation. A Comparison of biofilm formation ability of $P$. putida KT2440 in the presence of 4 different iron concentrations. B eDNA quantity in the presence of different iron concentrations. C

5 Comparison of PI-positive percentage among DGC, $P$. composti, $P$. putida KT2440 and $\Delta l a p A$ in 6 the presence of high $(100 \mu \mathrm{M})$ and low iron concentration $(5 \mu \mathrm{M})$. 
A

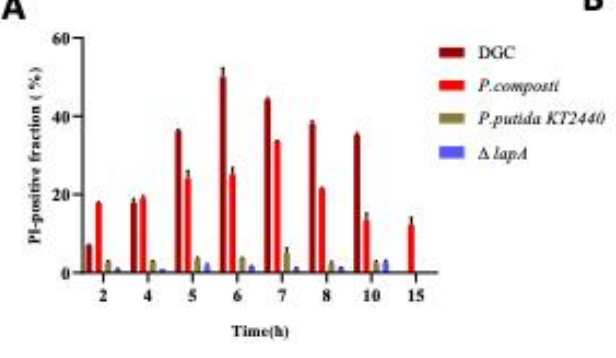

B

C

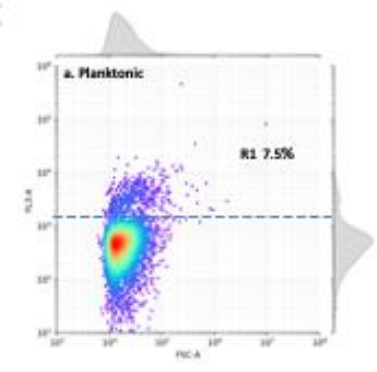

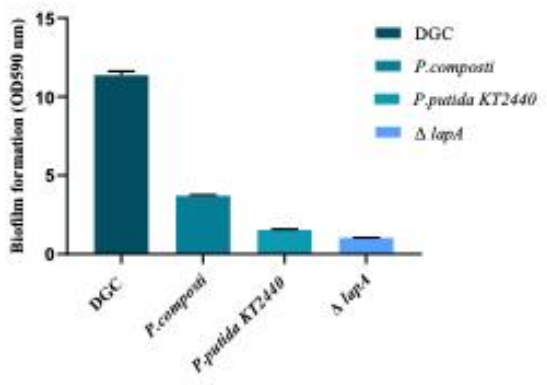
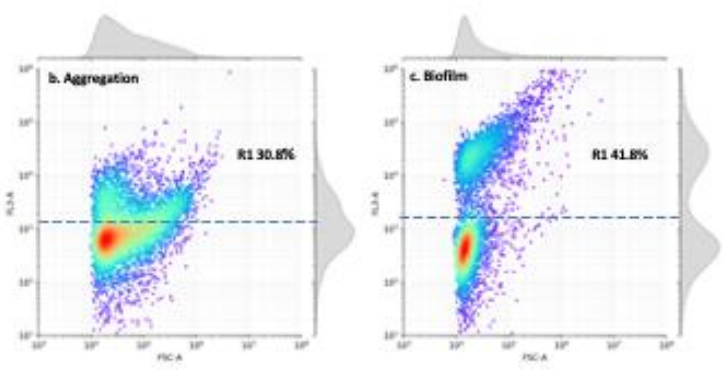

1

2 Figure 4. Comparison of PI-positive subpopulation percentage and biofilm formation capability 3 among DGC, P. composti, P. putida KT2440, $\Delta$ lapA. A Comparison of PI-positive fraction (\%)

4 cells during batch phase, $N \geq 3$. B Biofilm formation ability after $48 \mathrm{~h}, N \geq 5$. C Flow cytometry

5 comparison of PI-positive subpopulation for planktonic, aggregated and biofilm sample from DGC 6 strain. 


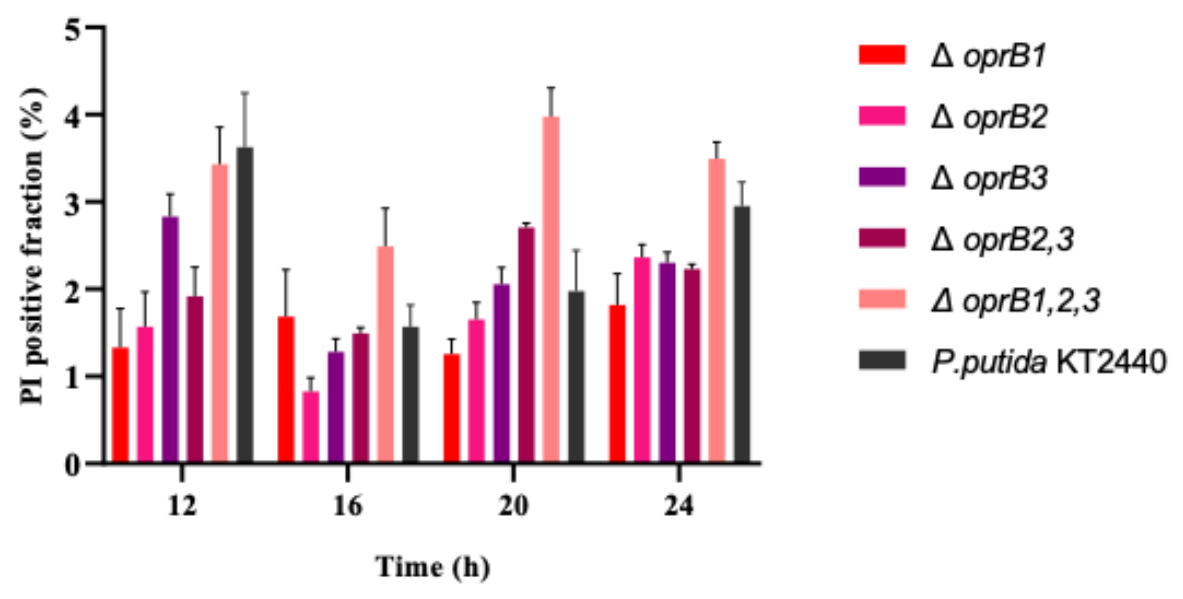

2 Figure 5. Effects of deletion porins group B on outer membrane permeability in P. putida KT2440 3 mutant, $N \geq 3$.

4

A

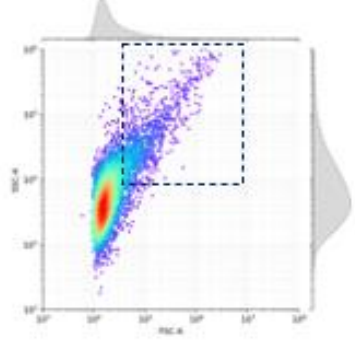

B
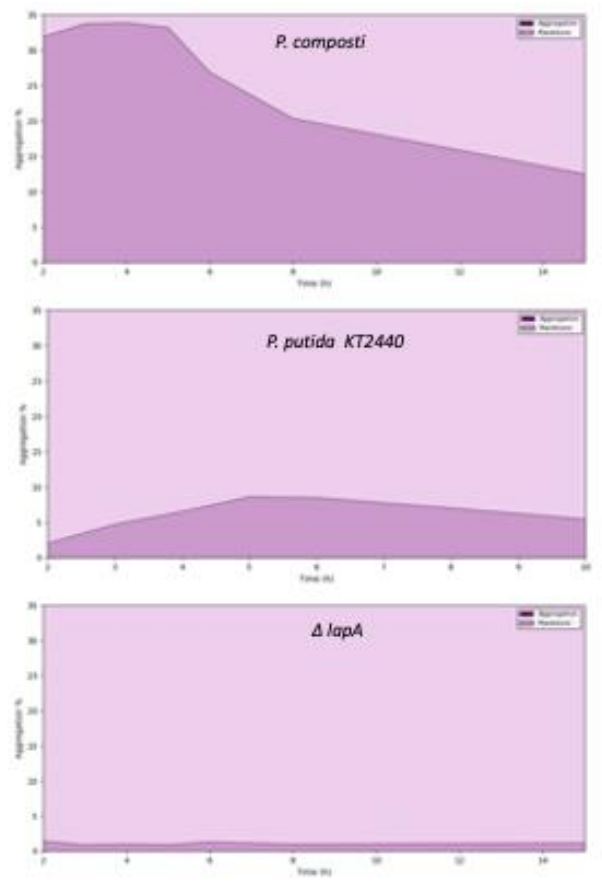

6 Figure 6. A Gating the aggregation subpopulation on flow cytometry based on FSC and SSC

7 signals. The blue dotted box separates the aggregation fraction from single cells. B Comparison of 
1 the degree of aggregation for $P$. composti, $P$. putida KT2440 and its $\Delta l a p A$ derivative by FC, $N \geq$ 22.
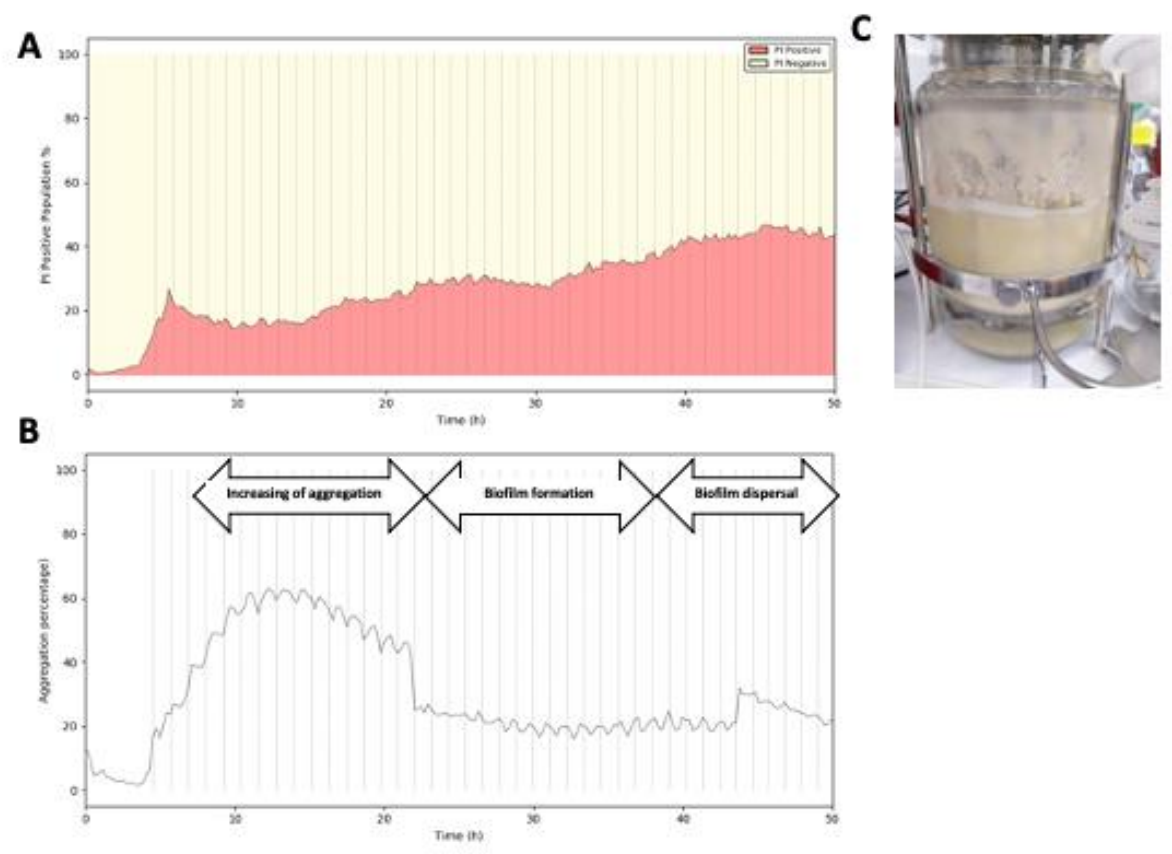

$5 \quad$ Figure 7. Dynamics of phenotypic diversification in pulsed continuous cultivation system in $P$. putida KT2440. A PI-positive dynamic in continuous feast and famine nutrient cycle during $50 \mathrm{~h}$. B Aggregation fluctuation dynamic during $50 \mathrm{~h}$ in automated pulsed continuous-culture bioreactor. C Macroscopic view of biofilm formation layer on the wall of the 2-L bioreactor after $50 \mathrm{~h}$ of 9 cultivation. 


\section{Supplemental information}

2

3 Table S1: comparison of auto-aggregation ability of different strains: P. putida DGC (KT2440

4 background), P. putida $\triangle$ lapA (KT2440 background), P. putida KT2440 and P. composti. Values in a

5 row that are not preceded by the same letter are significantly different $(p \leq 0.01)$

\begin{tabular}{|c|c|c|}
\hline Phenotypes & Strains & Autoaggregation (\%) \\
\hline $\begin{array}{c}\text { Agg+ } \\
\text { ( Strongly autoaggregation) }\end{array}$ & DGC & $100.00 \pm 0^{\mathrm{a}}$ \\
\hline $\begin{array}{c}\text { Agg- } \\
\text { (non-autoaggregation) }\end{array}$ & $\Delta$ lapA & $20.89 \pm 1.84^{\mathrm{d}}$ \\
\hline $\begin{array}{c}\text { Agg +/- } \\
\text { (Moderate autoaggregation) }\end{array}$ & P. putida KT2440 & $37.67 \pm 2.96^{\mathrm{c}}$ \\
\hline
\end{tabular}

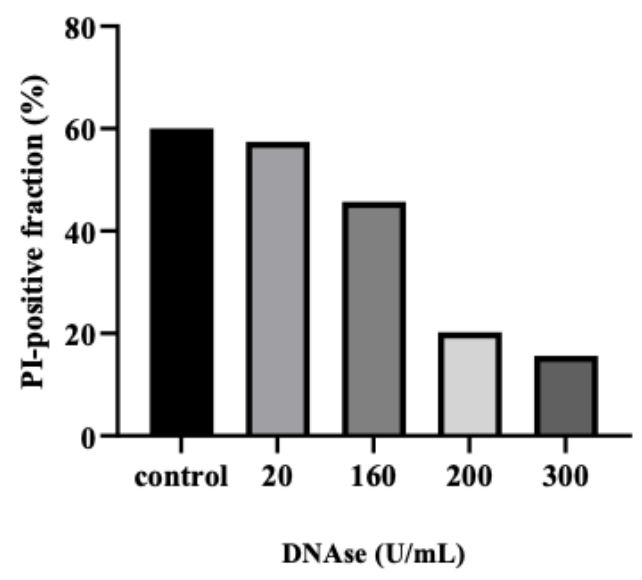

9

10 Figure S1: effect of different DNase I concentrations on the PI-positive fraction. 


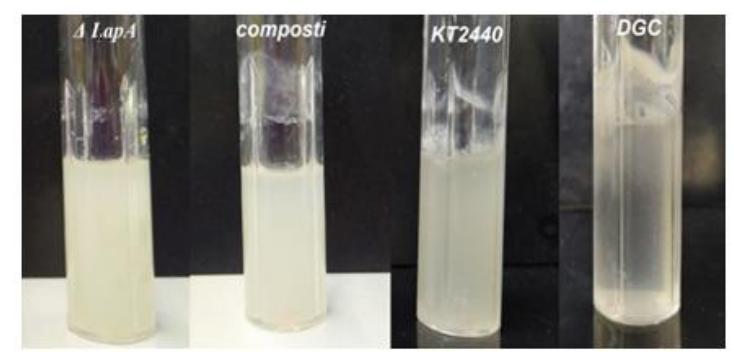

3 Figure S2: macroscopic view of biofilm formation on the wall of mini bioreactor vessel among $4 \Delta$ lapA, P. composti, P. putida KT2440 and DGC at the end of the batch phase.

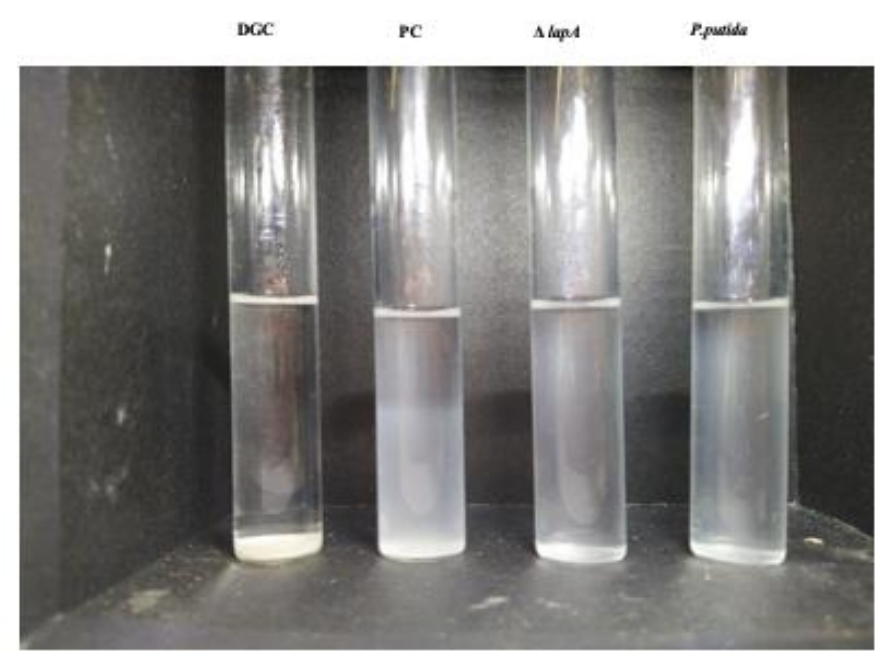

6 Figure S3: visual inspection the auto-aggregation ability of different strains: P. putida DGC (KT2440 7 background), P. putida $\triangle$ lapA (KT2440 background), P. putida KT2440 and P. composti. Auto8 aggregation ability has been determined over a period of $24 \mathrm{~h}$ at room temperature based on their 9 sedimentation characteristics 
4 Movie S1: evolution of PI-positive and aggregates subpopulations, based on individual dotplots 5 with forward scatter (FSC) as $x$-axis and PI fluorescence as $y$-axis. These dotplots were acquired 6 at an interval of 12 minutes by on-line flow cytometry and were further assembled into a single 7 .avi movie file

8 